\title{
Bending Strength Dependence of Fractoluminescence Intensity in Silica Glass, Single Crystal and Polycrystalline MgO
}

\author{
Tadashi Shiota ${ }^{*}$, Kouichi Yasuda and Yohtaro Matsuo \\ Department of Metallurgy and Ceramics Science, Graduate School of Science and Engineering, \\ Tokyo Institute of Technology \\ 2-12-1-S7-14, Ookayama, Meguro-ku, Tokyo 152-8552, Japan \\ *Corresponding author: tshiota@ceram.titech.ac.jp \\ ( Manuscript received 30 June 2008; accepted 21 August 2008; published 15 October 2008 ) \\ ( Presented at JAST Tribology Conference in Tokyo, May 2008)

\begin{abstract}
Fractoluminescence (FL) intensity and bending strength were simultaneously measured in silica glass, single crystal and polycrystalline $\mathrm{MgO}$ to study dependence of the FL on mechanical properties. In the silica glass, the FL intensity was positively correlated with the bending strength. Also, the positive correlation was found in the single crystal $\mathrm{MgO}$. However, there was not clear dependence in the polycrystalline $\mathrm{MgO}$. This suggests that the FL intensity is connected with the energy released by fracture in glasses and single crystal ceramics, and that grain boundary also contributes to the FL in polycrystalline ceramics.
\end{abstract}

Keywords: fractoluminescence, mechanical property, fracture, bending strength, silica glass, $\mathrm{MgO}$

\section{Introduction}

Luminous phenomena often accompany large earthquakes ${ }^{1,2)}$, and are associated with charged particle emission $^{3,4)}$ or luminescence ${ }^{5)}$ during fracture of rocks. Such particle emission during fracture of solids was referred as "fractoemission" and systematically studied first by Dickinson et al. ${ }^{6)}$ The photon emission during the fracture is often called "fractoluminescence" (FL), and many studies on the FL have been reported so far. The authors have also studied the FL in ceramics and glasses $^{7-10)}$. Although a lot of the FL mechanisms are proposed, for example, relaxation of color centers ${ }^{7,11-13)}$, gas discharge ${ }^{8,14)}$, thermal radiation ${ }^{7,14)}$ and so on, the mechanism is not completely elucidated yet. For an application of the FL to prediction of the earthquake or to evaluation of fracture behavior, it is necessary to elucidate the FL mechanism completely and then to establish a quantitative model for the FL.

An approach to elucidate the FL mechanism is to study a relation between the FL and mechanical properties of material. However, only a few experimental results have been reported on the relation ${ }^{13,15,16)}$. The purpose of this study is to clarify the relation in ceramics and glasses. In this study, silica glass, single crystal and polycrystalline $\mathrm{MgO}$ were selected as samples, because their mechanical properties are understood well and the FL on those materials was reported previously. The FL intensity and bending strength were simultaneously measured to study the relationship between them.

\section{Experimental}

Synthetic silica glass (Covalent Materials Co., $\mathrm{T}-4042$ ), single crystal MgO (Tateho Chemical Industries Co., Ltd., MIRACRYATAR) and polycrystalline $\mathrm{MgO}$ (Nikkato Co., MG-12G) were used as samples. The polycrystalline $\mathrm{MgO}$ had purity of $99.6 \%$, average grain size of $31 \mu \mathrm{m}$ and porosity of $7 \%$. Test specimens were fractured at room temperature by three-point bending at a crosshead speed of $0.1 \mathrm{~mm} / \mathrm{s}$. An applied load to the specimen was converted to an electrical signal using a load cell and amplifier, and recorded by a digital oscilloscope at a sampling time of $1 \mathrm{~ms}$. Bending strength was estimated from the equation,

$$
\sigma=\frac{3 P L}{2 w h^{2}}
$$

where $P, L, w$ and $h$ are the load at fracture, the support span of $16 \mathrm{~mm}$, width and thickness of the specimen, respectively. The FL was detected in two wavelength windows by two photomultiplier tubes (Hamamatsu Photonics, R2256P and R1221P) and optical filters. The FL intensity was measured by a photon counting method with multichannel scalar at a gate time of $1 \mathrm{~ms}$. Details of the experimental apparatus are described elsewhere ${ }^{9)}$. 
The silica glass was cut into a rectangular specimen with $3 \times 2 \times 20 \mathrm{~mm}^{3}$, and fractured at the pressure lower than $8 \times 10^{-5} \mathrm{~Pa}$. The FL in the silica glass was measured in the wavelength windows of $350 \sim 520 \mathrm{~nm}$ (VIS) and $600 \sim 850 \mathrm{~nm}$ (IR). These windows covered two emission bands of the FL in silica glass reported by Kawaguchi ${ }^{12)}$.

The single crystal and polycrystalline $\mathrm{MgO}$ were cut into $3 \times 1.6 \times 20 \mathrm{~mm}^{3}$ rectangular specimen. In the three-point bending test for the single crystal specimen, a crack propagation plane was set to be $\{100\}$, and the load was applied along to [100]. The FL in the single crystal and polycrystalline $\mathrm{MgO}$ was measured in 160 $650 \mathrm{~nm}$ (UV/VIS) and $640 \sim 850 \mathrm{~nm}$ (IR). The wavelength windows were also determined based upon the FL spectrum ${ }^{11)}$. The measurements were carried out in air with the humidity of $47 \sim 57 \%$. The effect of surrounding gases was also studied by the authors to confirm that the air did not greatly affect the FL in the $\mathrm{MgO}$ at atmospheric pressure ${ }^{8)}$.

\section{Results and discussion}

\subsection{Silica glass}

Figures 1 (a) to (c) show the result of simultaneous measurement of the applied load, VIS-FL and IR-FL, respectively. Both of the VIS-FL and IR-FL intensities were intensely increased at the moment of the fracture, and then decayed exponentially. The decay time of the VIS-FL was longer than that of the IR-FL. The FL decay does not directly reflect fracture process, that is, crack propagation process, because the specimens were fractured in an order of microsecond much faster than the gate time $(1 \mathrm{~ms})$. It is considered that the FL process involves electron transition from excited states to

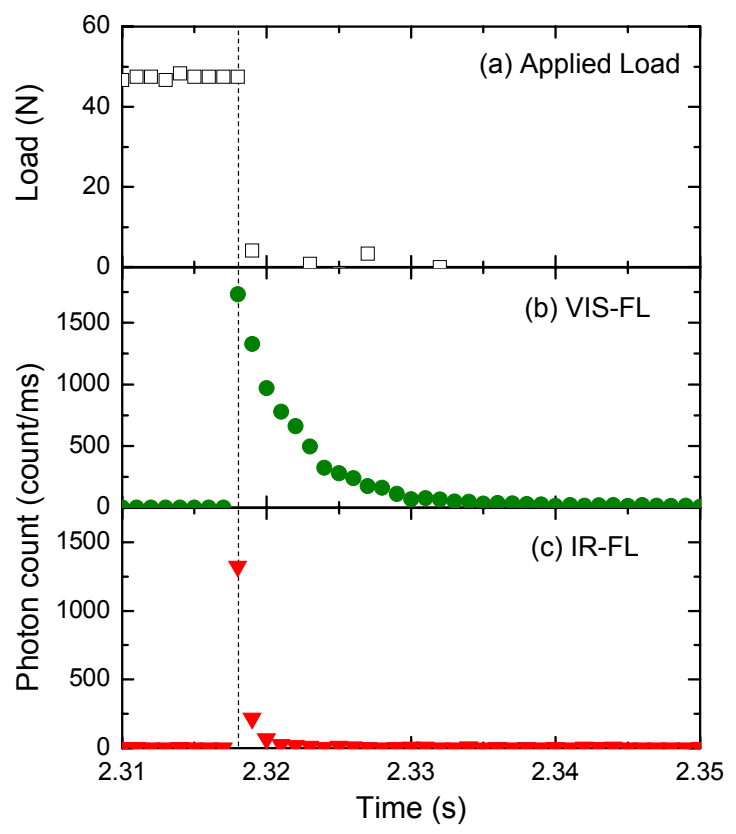

Fig. 1 Simultaneous measurement of (a) applied load, (b) VIS-FL and (c) IR-FL in silica glass

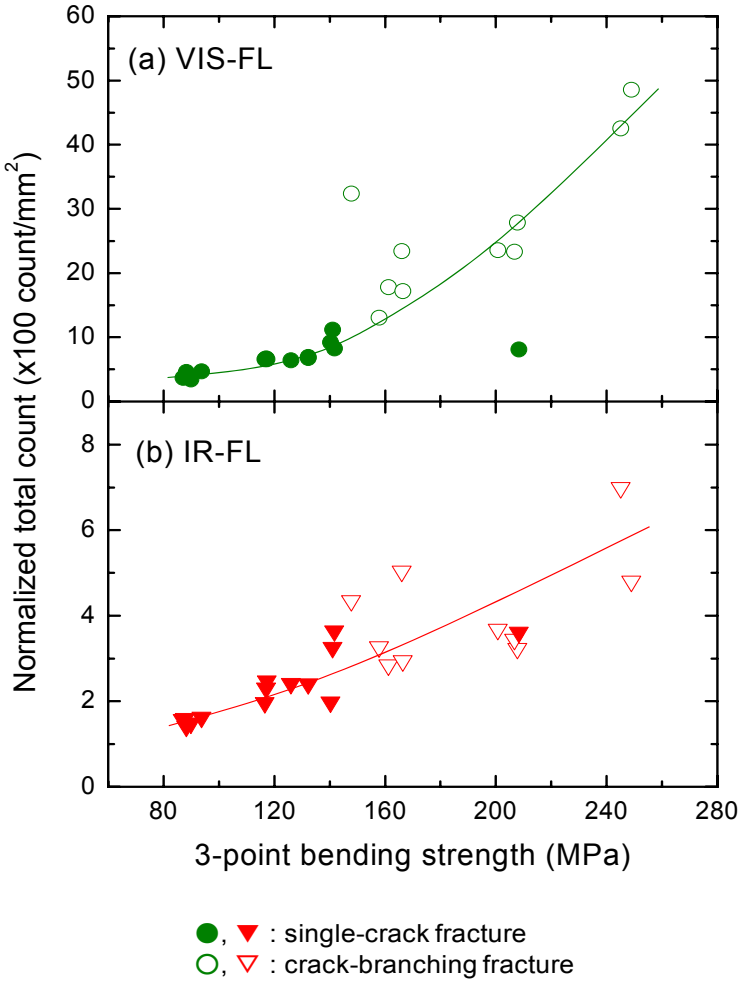

Fig. 2 Bending strength dependence of (a) VIS-FL and (b) IR-FL intensities in silica glass

ground states ${ }^{11,12)}$ and/or diffusion-related processes, for example, the recombination of electrons and holes ${ }^{13,17)}$. Therefore, the FL decay time probably depended on the lifetime of the excited electrons and/or the diffusion properties, and moreover the gate time of the measuring system might influence the measured decay curve.

The three-point bending test was made for 25 specimens to study a relationship between the FL intensity and the bending strength. Figures 2 (a) and (b) show dependence of the VIS-FL and IR-FL intensities on the bending strength, respectively. The FL intensity means the total count of the detected photons normalized by cross sectional area of the specimen before the fracture. The filled circles and triangles denote the data for the case that the specimen was fractured into two pieces, referred as "single-crack fracture" hereafter. The open circles and triangles denote the data for the case that the specimen was fractured into more than three pieces, referred as "crack-branching fracture". It was found that both of the VIS-FL and IR-FL intensities were positively correlated with the bending strength in silica glass.

The correlation was nonlinear, which was different from that in the single crystal $\mathrm{MgO}$ as described in the next section. The FL intensity is proportional to the total number of FL centers which is determined by density of the FL centers and emitting surface area. Since the energy released by fracture is increased with bending strength, the positive correlation is ascribed to the increase in the FL center density and/or the emitting 


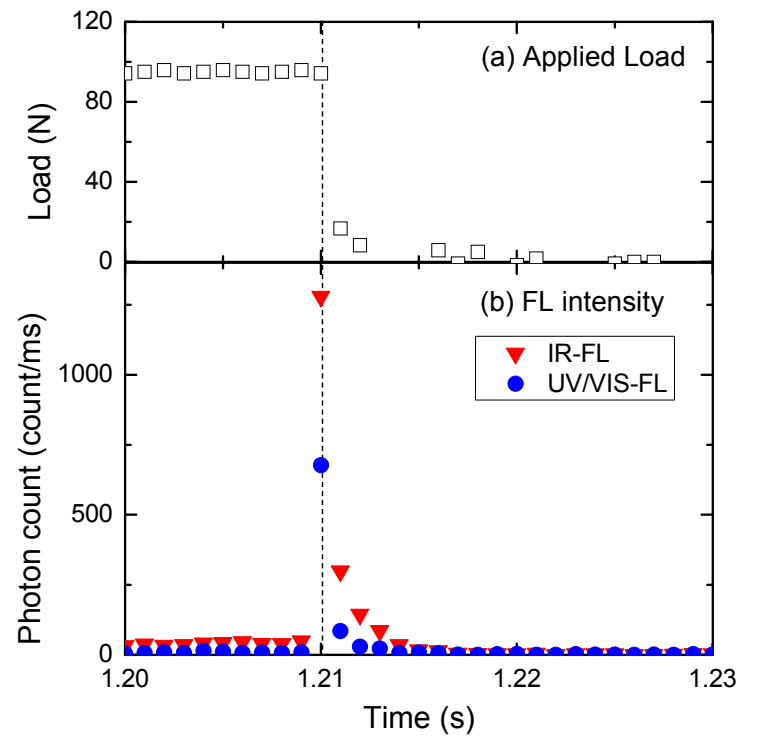

Fig. 3 Time evolution of (a) applied load and (b) FL intensity in single crystal $\mathrm{MgO}$

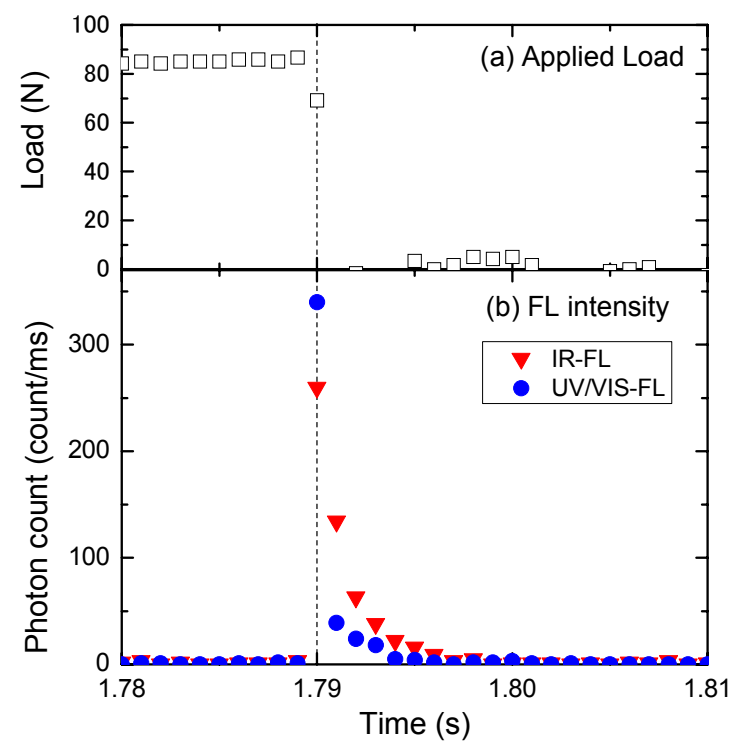

Fig. 4 Time evolution of (a) applied load and (b) FL intensity in polycrystalline $\mathrm{MgO}$

surface area with the released energy. In the single-crack fracture, it is probable that the positive correlation resulted from the increase in the FL center density because the emitting surface area was almost constant. Thus, the FL center density depends on the released energy, which must be the case for the crack-branching fracture. In addition, the crack branching also caused the increase in the emitting surface area. This may be the reason why the FL intensity was increased nonlinearly with the bending strength.

\subsection{Single crystal and polycrystalline $\mathrm{MgO}$}

Figures 3 and 4 show the FL in the single crystal and polycrystalline $\mathrm{MgO}$, respectively. In each figure, the applied load is shown in (a), and the UV/VIS-FL and IR-FL intensities are shown in (b) by filled circles and (a) UVNVIS-FL

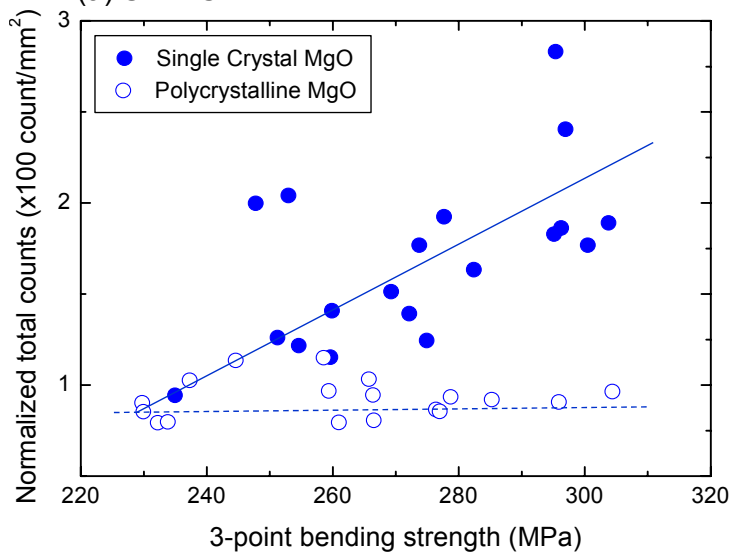

(b) IR-FL

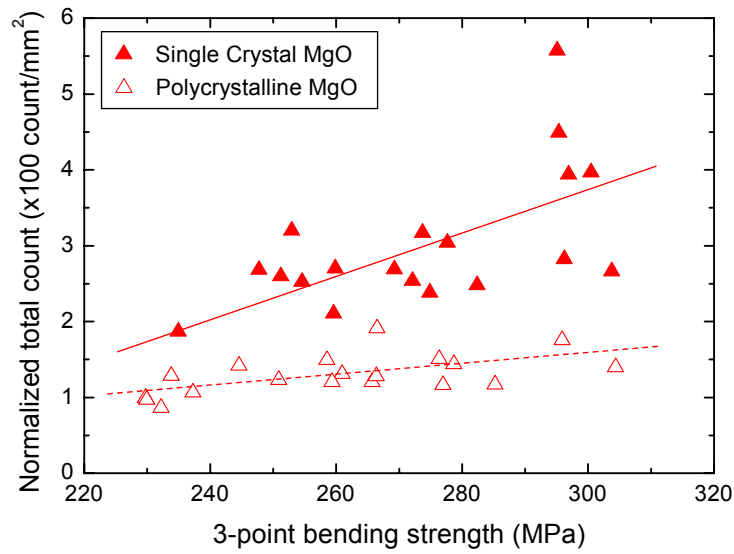

Fig. 5 Bending strength dependence of (a) UV/VIS-FL and (b) IR-FL intensities in single crystal and polycrystalline $\mathrm{MgO}$

triangles, respectively. Both of the UV/VIS-FL and IR-FL characteristics were similar to those of the silica glass as shown in Fig. 1. Note that there was not clear difference in the decay time of the UV/VIS-FL and IR-FL, different from the FL on silica glass. The difference in the FL decay between $\mathrm{MgO}$ and silica glass may be attributed to the lifetime of the excited electrons at each FL center, the diffusion properties and /or the gate time, as described in the previous section.

In order to make clear a relationship between the FL intensity and the bending strength in the $\mathrm{MgO}$, the three-point bending test was repeated for 19 single crystal specimens and for 18 polycrystalline specimens. Figures 5 (a) and (b) show the results for the UV/VIS-FL and IR-FL, respectively. The UV/VIS-FL intensity in the single crystal $\mathrm{MgO}$ was positively correlated with the bending strength, whereas there was not clear dependence in the polycrystalline $\mathrm{MgO}$. This was also the case for the IR-FL.

All the single crystal specimens exhibited the single-crack fracture. Namely, the increase in the FL center density with the released energy contributed to the positive correlation. Dickinson et al. observed strong photoluminescence (PL) from step edges and deformed 
areas on a cleaved surface of single crystal $\mathrm{MgO}^{18)}$. It was reported that both of the PL and FL were originated from the same emission centers such as $F$ or $\mathrm{F}^{+}$ centers $^{7,11,13)}$. Therefore, the positive correlation in the single crystal $\mathrm{MgO}$ was attributed to the increase in the step density and deformed area on the fracture surface with increasing the released energy by the fracture.

In the case of the polycrystalline $\mathrm{MgO}$, the UV/VIS-FL and IR-FL intensities did not clearly depend on the bending strength. All the polycrystalline specimens also showed the single-crack fracture. When polycrystalline ceramics is fractured, a crack propagation behavior becomes complex because of the presence of grain boundaries. Consequently, the polycrystalline ceramics exhibits a mixed mode of transgranular fracture and intergranular fracture, which is different from fracture behavior in glasses and single crystal ceramics. The authors reported that the FL intensity was increased with a proportion of the transgranular fracture in polycrystalline $\mathrm{MgO}$ and $\mathrm{Al}_{2} \mathrm{O}_{3}{ }^{7)}$. Therefore, due to the effect of grain boundary, no clear correlation between the FL intensity and the bending strength may be observed in the polycrystalline $\mathrm{MgO}$. Further study is required to clarify the relation among the FL intensity, the bending strength and the presence of grain boundary.

\section{Conclusion}

The FL intensity and bending strength were simultaneously measured in silica glass, single crystal and polycrystalline $\mathrm{MgO}$ to study a relation between the FL and mechanical properties. In the case of the silica glass and single crystal $\mathrm{MgO}$, the $\mathrm{FL}$ intensity was positively correlated with the bending strength. However, there was not clear dependence in the polycrystalline $\mathrm{MgO}$, which was caused by a complex behavior of crack propagation due to grain boundaries. This study suggests that the strength data is useful for analysis of the FL in glasses and single crystal ceramics. In addition, grain boundaries should be taken into account for the FL analysis in polycrystalline ceramics.

\section{References}

[1] Derr, J. S., "Earthquake Lights: A Review of Observations and Present Theories," Bulletin of the Seismological Society of America, 63, 1973, 2177-2187.

[2] Kamogawa, M., Ofuruton, H. and Ohtsuki, Y., "Earthquake Light: 1995 Kobe Earthquake in Japan," Atmospheric Research, 76, 2005, 438-444.

[3] Enomoto, Y. and Hashimoto, H., "Emission of Charged Particles from Indentation Fracture of Rocks," Nature, 346, 1990, 641-643.

[4] Enomoto, Y., Akai, M., Hashimoto, H., Mori, S. and Asabe, Y., "Exoelectron Emission: Possible Relation to Seismic Geo-Electromagnetic Activities as a Microscopic Aspect in Geotribology," Wear, 168, 1993, 135-142.
[5] Derr, J. S., "Luminous Phenomena and Their Relationship to Rock Fracture," Nature, 321, 1986, 470-471.

[6] Dickinson, J. T., Donaldson, E. E. and Park, M. K., "The Emission of Electrons and Positive Ions from Fracture of Materials," Journal of Materials Science, 16, 1981, 2897-2908.

[7] Yasuda, K., Shimada, M. and Matsuo, Y., "Some Aspects of Photon Emission of Polycrystalline Ceramics during Fracture," Philosophical Magazine A, 82, 17\&18, 2002, 3251-3261.

[8] Shiota, T., Toyoshima, Y., Yasuda, K. and Matsuo, Y., "Gas Pressure Dependence of Photon Emission Accompanying Fracture of Polycrystalline $\mathrm{MgO}$ in Nitrogen," Key Engineering Materials, 317-318, 2006, 313-316.

[9] Shiota, T., Yasuda, K. and Matsuo, Y., "Correlation between the Flexure Strength and the Photon Emission Intensity during Fracture on Single Crystal and Polycrystalline MgO," Materials Science and Engineering: B, 148, 2008, 230-233.

[10] Shiota, T., Yasuda, K. and Matsuo, Y., "Dependence of the Particle Emission during the Fracture of Silica Glass on Its Mechanical Property," Journal of Physics: Conference Series, 100, 2008, 072041.

[11] Kawaguchi, Y., "Luminescence Spectra at Bending Fracture of Single Crystal MgO," Solid State Communications, 117, 2001, 17-20.

[12] Kawaguchi, YTime-Resolved Fractoluminescence Spectra of Silica Glass in a Vacuum and Nitrogen Atmosphere," Physical Review B, 52, 13, 1995, 9224-9228.

[13] Langford, S. C., Dickinson, J. T. and Jensen, L. C., "Simultaneous Measurements of the Electron and Photon Emission Accompanying Fracture of Single-Crystal MgO," Journal of Applied Physics, 62, 1987, 1437-1449.

[14] Brady, B. T. and Rowell, G. A., "Laboratory Investigation of the Electrodynamics of Rock Fracture," Nature, 321, 1986, 488-492.

[15] Krell, A., "Fracture-Induced Photon Emission of Aluminum Oxide and Its Application to Fracture Mechanical Investigations," Journal of Materials Science, 17, 1982, 1649-1655.

[16] Mizuno, Y. and Mizuno, T., "Photon Emission from Ice during Fracture," Japanese Journal of Applied Physics, 41, Pt. 2, 2002, L209-L211.

[17] Dickinson, J. T., Langford, S. C., Jensen, L. C., McVay G. L., Kelso J. F., Pantano C. G., "Fractoemission from Fused Silica and Sodium Silicate Glasses," Journal of Vacuum Science and Technology A, 6, 1988, 1084-1089.

[18] Dickinson, J. T., Jensen, L. C., Webb, R. L. and Langford, S. C., "Photoluminescence Imaging of Mechanically Produced Defects in MgO," Journal of Non-Crystalline Solids, 177, 1994, 1-8. 